\title{
Bienestar en personas mayores en situación de pobreza: determinantes y significados
} Well-being of older adults in poverty: Determinants and meanings

\author{
María Fernanda Reyesa, Paula Altamarb, Martha Aguirrea y Diana Murilloa \\ aUniversidad El Bosque, Bogotá, Colombia ${ }^{b}$ Facultad Latinoamericana de Ciencias Sociales, México D. F., México
}

\begin{abstract}
Resumen
Se realizó una investigación cualitativa de carácter exploratorio con el objetivo de identificar los determinantes de bienestar subjetivo en personas mayores en situación de pobreza. Se analizaron 12 entrevistas de hombres y mujeres con edades entre los $60 \mathrm{y}$ 80 años. El análisis de los datos se llevó a cabo mediante la teoría fundamentada, y se encontró que el bienestar subjetivo en las personas mayores está determinado por factores como: percepción de control, apoyo social, vinculación a actividades productivas, seguridad económica, salud, factores culturales, fortalezas personales y oportunidad de acceder a información y capacitación. Con estos hallazgos se construyó un modelo en donde se definen las relaciones entre las categorías y subcategorías.
\end{abstract}

Palabras clave: bienestar subjetivo, personas mayores, teoría fundamentada.

\begin{abstract}
An exploratory qualitative research was conducted aiming to identify the determinants of subjective well-being of older adults living in poverty. A total of 12 semi-structured interviews were conducted in a sample of men and women from 60 to 80 years old. Data were analyzed using the grounded theory approach, and the results showed that subjective well-being in older adults is determined by: perceived self-control, social support, linkage to productive activities, economic security, health, cultural factors, personal strengths and opportunity to access information and training. With this findings, a model that defines the relationships between the categories and sub-categories was built.
\end{abstract}

Keywords: subjective well-being, older adults, grounded theory.

Contacto: M. F. Reyes Rodríguez. Facultad de Psicología. Universidad El Bosque. Av. Cra 9 No. 131 A - 02, Edificio Fundadores, Piso 2. Bogotá, Colombia. reyesmariafr@unbosque.edu.co

Cómo citar este articulo:

Reyes, M. F., Altamar, P., Aguirre, M. y Murillo, D. (2014). Bienestar en personas mayores en situación de pobreza: determinantes y significados. Revista de Psicología, 23(2), 101-115.

http://dx.doi.org/10.5354/0719-0581.2014.36151 


\section{Introducción}

El rápido envejecimiento de la población es un fenómeno mundial, el cual según el reporte del Fondo de Poblaciones de las Naciones Unidas en el 2012, está sucediendo de manera más rápida en países en desarrollo (United Nations Population Fund [UNFPA], 2012). Colombia a pesar de seguir siendo un país joven, está experimentando estos cambios, pues la población de 60 años y más, era de cerca del 6\% en el 2007, y de $10 \%$ en el 2010 y se estima que el 2050 sea de cerca del 21\% (Profamilia, 2010; Secretaría Distrital de Integración Social [SDIS], 2008). Bogotá también ha registrado un crecimiento del $5.3 \%$ de la población de 60 años y más en los últimos 15 años; actualmente cerca de 618 mil personas tienen más de 60 años (Alcaldía Mayor de Bogotá, 2010).

Las condiciones de vida de las personas mayores en Colombia son preocupantes, pues solo el $25 \%$ recibe algún tipo de pensión por jubilación ${ }^{1}$ y se estima que cerca del 40\% de las personas envejece en condiciones de pobreza (Profamilia, 2010). En Bogotá menos del $40 \%$ de las personas mayores recibe una pensión por jubilación (Universidad Nacional de Colombia, Fondo de Población de las Naciones Unidas y SDIS, 2009), y se estima que existen 19879 hogares con necesidades básicas insatisfechas donde viven personas de 60 años y más (Alcaldía Mayor de Bogotá, 2010). Estos indicadores han generado iniciativas para la atención a esta población, las cuales, desafortunadamente, siguen siendo insuficientes.

Las políticas públicas suponen estar orientadas a garantizar el bienestar de la población, sin embargo, actualmente no se cuenta con un modelo que facilite la comprensión del constructo "bienestar en la vejez" adaptado a la realidad colombiana, pues comúnmente se usan modelos de bienestar construidos en países desarrollados los cuales no necesariamente aplican para países en

${ }^{1}$ En Colombia se pensionan solo las personas que han cotizado al sistema de seguridad social, durante aproximadamente 1000 semanas, y cumplen con la edad de 55 a 57 años para las mujeres y 60 a 62 años para los hombres. Las personas que no cumplen estos criterios no se pensionan.

Revista de Psicología

2014, 23(2), 101-115 desarrollo y/o a la población mayor en situación de pobreza.

Esta situación ha generado la necesidad de desarrollar estándares, conceptos, teorías y prácticas propias, que respondan al contexto social y cultural y no a la imposición de ideas de países desarrollados (Teo, 2009). Por esta razón, se requiere comprender los factores que se relacionan con el bienestar de las personas mayores colombianas en situación de pobreza, para contribuir a estos desarrollos.

El estudio del bienestar ha tenido tradicionalmente dos miradas: el hedonismo y la eudamoinia. La primera se enfoca en el placer y felicidad, mientras que la segunda, considera que el bienestar va más allá de la felicidad, pues es el resultado de un proceso y no es solo situacional (Ryan y Deci, 2001). Esta última perspectiva considera el bienestar como la evaluación subjetiva que hacen las personas de sí mismos, a nivel general, el grado en que las personas experimentan un sentido de bienestar (Deci y Ryan, 2008).

El presente estudio concibe el bienestar subjetivo (BS) como la percepción que las personas tienen sobre sus vidas, relacionado con sus pensamientos y sentimientos; así como las conclusiones que se derivan de evaluar su existencia (Diener, Eunkook, Richard, Lucas y Smith, 1999). El BS está compuesto por dos facetas, una se centra en los aspectos afectivoemocionales y la otra en los aspectos cognoscitivo-valorativos. Debe contener siempre tres componentes: (a) satisfacción con la vida, (b) afecto positivo y (c) afecto negativo (Diener et al., 1999; Diener, Oishi y Lucas, 2003).

El bienestar ha sido de gran interés para diversas disciplinas psicológicas, sociales y económicas (Deci y Ryan, 2008; Pethtel y Chen, 2010). Desde la psicología se han diferenciado dos grandes perspectivas: (a) bienestar subjetivo y (b) bienestar psicológico. Estos dos conceptos se relacionan y comparten dimensiones, sin embargo, se conciben como diferentes. El bienestar subjetivo se refiere a una evaluación global de la vida, mientras que el bienestar psicológico se refiere especialmente a la evaluación del cumplimiento de los propósito en la vida (Keyes, Shmotkin y Ryff, 2002). Este estudio se centra solo en el BS. 
El BS tiene varios componentes interrelacionados (Panel on Measuring Subjective Well-Being in a Policy-Relevant Framework, Committee on National Statistics, Division on Behavioral and Social Sciences and Education y National Research Council, 2013). Así, el bienestar subjetivo está determinado por factores externos e internos, como la personalidad, cogniciones, aspectos demográficos, sociales y culturales (Deci y Ryan, 2008; Diener et al., 1999).

Dentro de los factores internos está la percepción de control (dominio del ambiente), que se ha identificado como factor fundamental para el BS en la vejez, pues en esta etapa existe una alta probabilidad de enfrentar situaciones que amenazan el control del ambiente (Cárdenas y López, 2010; Daatland y Hansen, 2007; Ong y Bergeman, 2004). Otros determinantes del bienestar serían la salud objetiva y subjetiva (Fernández-Ballesteros, 2009; Ryff y Keyes, 1995), y la realización de actividades que sean valoradas como productivas para las personas mayores, es decir, que tengan un significado motivacional (Fernández-Ballesteros, 2009; Mella et al., 2004).

Por otra parte, se han identificado algunos recursos personales que están relacionados con una percepción de bienestar, algunos rasgos de personalidad (e.g., extroversión y apertura a la experiencia), la autoestima y la autoeficacia (Benight y Bandura, 2004; Ouwehand, De Ridder y Bensing, 2007; Windle y Woods, 2004). Por ejemplo, existe evidencia de que las personas extrovertidas manifiestan mayor afecto positivo (Diener et al., 2003) y que las personas resilientes y optimistas reportan un mayor BS que quienes no lo son (Ong, Bergeman y Boker, 2009).

Uno de los factores que ha sido más estudiado como generador de bienestar es el apoyo social (Bisconti y Bergeman, 1999; Hildon, Montgomery, Blane, Wiggins y Netuveli, 2010; Merz y Huxhold, 2010) pues facilita la satisfacción de necesidades básicas, informativas y emocionales (Vera, Sotelo y Domínguez, 2005), y promueve los vínculos entre personas en realización de actividades productivas (remuneradas y no remuneradas) (Bisconti y Begerman, 1999), lo que se asocia con una mayor satisfacción con la vida, felicidad y autoestima (Fernández-Ballesteros, 2009).
Respecto al tipo de redes de apoyo, se ha encontrado que no solo la familia y amigos son una fuente de apoyo social, sino que el Estado juega un papel importante como fuente de apoyo en la vejez (Requena, 2003). Otro de los aspectos fundamentales es el factor económico, debido a que los ingresos pueden garantizar acceso a vivienda, lo que ha sido identificado como crucial para el bienestar (Windle y Woods, 2004).

En Latinoamérica uno de los pocos estudios en la temática es el de Mella et al. (2004) quienes proponen un modelo predictivo para el BS de las personas mayores chilenas, sugiriendo que este se predice por la salud percibida, el apoyo social percibido, el sexo y la ausencia de depresión. Sin embargo, ese estudio evalúa solo un componente del bienestar, la satisfacción con la vida, y no aborda el aspecto afectivo, sus dimensiones ni sus factores asociados o relacionados. En Colombia, los estudios que existen están orientados a evaluar las percepciones de bienestar y envejecimiento (e.g., Alonso et al., 2010), mas no a generar modelos que permitan comprender los factores determinantes del bienestar.

Ahora bien, en el contexto latinoamericano los modelos de bienestar subjetivo en contextos de pobreza son limitados y podrían ser considerados como descriptivos. Por ejemplo, PonceZubillaga (2013) considera que es necesario estudiar el bienestar en un contexto de pobreza, porque puede llegar a tener implicancias positivas a nivel de política pública. Asimismo, la diversidad y multidimensionalidad del constructo, especialmente en un contexto de pobreza, hacen inminente la necesidad de comprenderlo.

El presente estudio tiene como objetivo conocer los factores que están asociados con el bienestar en la vejez en situación de pobreza, a través de una metodología cualitativa que permita generar un modelo con implicancias teóricas, sin pretender probar uno ya existente, sino construyendo uno propio reconociendo los factores psicológicos, sociales y culturales que lo determinan.

\section{Método}

Este estudio cualitativo de carácter exploratorio se realizó a través de la teoría fundamentada. Se realizaron 12 entrevistas semiestructuradas a 
personas mayores durante el año 2009 y en ellas se exploran las percepciones de bienestar subjetivo en un contexto de pobreza.

Los participantes del estudio pertenecían al proyecto "Atención Integral para la Garantía de los Derechos para una Vejez Digna en el Distrito Capital" de la Alcaldía de Bogotá, que tiene por objetivo "contribuir a la reducción de la discriminación por edad y la segregación socioeconómica de las personas mayores en la ciudad de Bogotá" (SDIS, 2012, p. 16). El proyecto ofrece servicios a personas de 60 años y más que se encuentren en condiciones de vulnerabilidad. Los servicios que ofrecen están dirigidos al desarrollo de capacidad, oportunidades y potencialidades a través de hogares gerontológicos, centros de día y noche, y apoyo económico mediante un subsidio asistencial (Departamento Administrativo de Bienestar Social [DABS], 2006; SDIS, 2008, 2012).

El año 2009, dicho subsidio consistía en el equivalente a 40 dólares americanos mensuales, y para recibirlo o encontrarse en lista de espera, las personas mayores no debían estar recibiendo ninguna pensión (no cotizaron las semanas necesarias en el sistema de seguridad social para poder pensionarse), estar clasificado como población vulnerable por el Sistema de Identificación y Clasificación de Potenciales Beneficiarios de los programas sociales (Sisben), tener un ingreso mensual menor al salario mínimo legal vigente (aproximadamente USD \$ 300) y no contar con un lugar en donde vivir (SDIS, 2008). El proyecto alcanza una cobertura del $20 \%$ de la población que cumple los requisitos para recibir los beneficios (SDIS, 2008).

\section{Participantes}

En el estudio participaron 12 personas de 60 años y más $(M=70$ años, $D E=7)$, cuatro hombres y ocho mujeres, pertenecientes al proyecto "Atención Integral para la Garantía de los Derechos para una Vejez Digna en el Distrito Capital" en la modalidad de apoyo económico. Del total de entrevistados, ocho se encontraban recibiendo un subsidio mensual equivalente a 40 dólares, tres estaban en lista de espera para recibir el subsidio y una persona era pensionada con un salario mínimo (USD\$ 300) que participaba en las actividades recreativas del proyecto pero no recibía el subsidio (Ver tabla 1). Las personas que están pensionadas pueden participar en las actividades recreativas del proyecto, pero no reciben ningún beneficio económico.

El enfoque de capacidades de Sen (1995) se tomó como base para determinar la selección de participantes. El enfoque de Sen se refiere a la evaluación comparativa del bienestar y justicia social, teniendo en cuenta las funciones y capacidades de las personas; busca responder la pregunta respecto a qué es capaz cada persona de ser y hacer, considerando a cada persona como un fin, sin necesariamente interesarse en el bienestar promedio, sino en las oportunidades de cada persona. Este enfoque considera que los logros y oportunidades que son importantes para las personas son diferentes en calidad y no solo en cantidad y, por ello, no se deben reducir a un número, siendo fundamental para entender el bienestar y generarlo, entender la naturaleza específica de cada uno (Nussbaum, 2011; Nussbaum y Sen, 1993). Sen (1995) argumenta que para el estudio del bienestar en un contexto de pobreza es fundamental tener en cuenta las funciones y capacidades de los individuos (Nussbaum y Sen 1993).

La presente investigación basó la selección de sus participantes en la evaluación comparativa que propone el enfoque de capacidades de Amartya Sen; por esto se eligieron aquellos perfiles con mayores diferencias entre sí y aquellos más cercanos al promedio. Para ello, se realizó un análisis de componentes principales para datos categóricos (CATPCA) con la base de datos completa 2008, del proyecto "Atención Integral para la Garantía de los Derechos para una Vejez Digna en el Distrito Capital" (Altamar, 2010), la cual contaba con 56440 personas registradas, de las cuales 38406 estaban recibiendo subsidio y 18034 estaban en lista de espera. Se excluyeron las personas menores de 60 años que estaban registradas como jubiladas (Altamar, 2010).

El CAPTCA, se utiliza para reducir una gran cantidad de datos categóricos en componentes 
no correlacionados que representen la mayoría de los datos (Linting, Meulman, Groenen y van der Kooij, 2007). Cada variable fue trabajada en función de su nivel de medición mediante el método de escalamiento óptimo, que permitió extraer los componentes que mejor resumen las relaciones observadas entre las variables. El análisis se realizó con las variables: sexo, edad, estado civil, priorización por condición económica, presencia de discapacidad, actividad remunerada, parentesco con el jefe de hogar, víctima de violencia intrafamiliar, relación con los hijos, aporte a pensión, actividad que realiza y puntaje Sibsen (Altamar, 2010).

Se definieron por saturación ocho dimensiones, cada una presenta una contribución diferente a la explicación de la varianza y, a su vez, las dimensiones se caracterizan de ciertas variables y categorías que jalonan esta diferencia. Las dimensiones se describen a continuación (Altamar, 2010).

Dimensión uno. Explica un 14.3\% de la variabilidad. Así, se definieron dos primeros grupos: aquellos que viven en pareja, son el cónyuge, hombres y se encuentran en un rango de edad de 60-69 años; y aquellas personas que son viudas, mujeres, se relacionan con el jefe de familia en calidad distinta a cónyuge o padre o madre y es nivel 3 de Sisben.

Dimensión dos. Explica un 13.6\% de la variabilidad. Se identifican dos grupos: personas que no trabajan, no se relacionan con sus hijos y son mujeres; y personas que trabajan, se relacionan con sus hijos y son hombres.

Dimensión tres. Explica un 10.6\% de la variabilidad. Analizando por categorías, se generaron dos grupos: uno constituido por los jefes de hogar que son hombres y altamente vulnerables al ubicarse en el nivel 0 y 1 del puntaje Sisben y, otro grupo, que son personas de nivel 3 y cónyuges.
Dimensión cuatro. Explica un $9.4 \%$ de la variabilidad. Por categorías se conforma un único grupo constituido por personas mayores víctimas de violencia intrafamiliar y que presentan discapacidad.

Dimensión cinco. Explica un $9.3 \%$ de la variabilidad. Las variables de apoyo económico y nivel Sisben son las que participan en mayor medida.

Dimensión seis. Explica un 9\% de la varianza. Entre las categorías, la variabilidad responde a personas que reciben apoyo económico en tensión con aquellas pensionadas.

Dimensión siete. Explica un $8.9 \%$ de la variabilidad. Esta dimensión es explicada únicamente por la variable de apoyo económico respecto al promedio de las personas mayores.

Dimensión ocho. Explica un 8.56\% de la varianza. Las variables que mejor explican la varianza son las mismas que en la dimensión cuatro, la discapacidad y la violencia intrafamiliar. Este punto, se asume como saturación debido a que no aporta mayor información para la construcción de un grupo nuevo, por lo cual se detiene el análisis por dimensiones.

La muestra para el presente estudio resultó en 12 personas mayores que cubren las características de los perfiles propuestos por las ocho dimensiones. Se incluyeron más personas con el fin de cumplir con las variables propuestas en los perfiles, especialmente aquellos en donde no fue posible cumplir con todas las características y para poder tener mayor variabilidad en lo perfiles de los participantes. En la tabla 1 se describen los perfiles de las personas seleccionadas.

Es importante resaltar que la mayoría de la población del proyecto eran mujeres, lo que explica por qué hay más mujeres que hombres en esta investigación. No se tuvo como fin comparar hombres y mujeres. 
Tabla 1

Características Sociodemográficas de los participantes

\begin{tabular}{|c|c|c|c|c|c|c|}
\hline Participante & Sexo & Edad & $\begin{array}{c}\text { Estado } \\
\text { civil }\end{array}$ & $\begin{array}{c}\text { Enfermedad / } \\
\text { Discapacidad } \\
\end{array}$ & Actividad actual & $\begin{array}{c}\text { Estado } \\
\text { proyecto }\end{array}$ \\
\hline Sr. C & $\mathrm{H}$ & 69 & Casado & No & Líder comunitario & Activo $^{a}$ \\
\hline Sra. MC & $\mathrm{F}$ & 84 & Viuda & $\begin{array}{c}\text { Sí } \\
\text { (Enfermedad de } \\
\text { Parkinson) }\end{array}$ & Ninguna & Activo $^{a}$ \\
\hline Sra. B & $\mathrm{F}$ & 69 & Soltera & No & Actividades recreativas & Activo $^{a}$ \\
\hline Sra. A & $\mathrm{F}$ & 60 & Soltera & $\begin{array}{c}\text { Sí } \\
\text { (Discapacidad } \\
\text { auditiva) }\end{array}$ & $\begin{array}{l}\text { Trabajo ocasional co- } \\
\text { mo aseadora }\end{array}$ & Activo $^{a}$ \\
\hline Sr. R & M & 68 & Casada & No & $\begin{array}{l}\text { Trabajo ocasional } \\
\text { obrero }\end{array}$ & Activo $^{a}$ \\
\hline Sra. BI & $\mathrm{F}$ & 65 & Casada & No & Actividades recreativas & Activo $^{a}$ \\
\hline Sra. V & $\mathrm{F}$ & 70 & Soltera & $\begin{array}{c}\text { Sí } \\
\text { (Ceguera) }\end{array}$ & Ninguna & Activo $^{a}$ \\
\hline Sra. H & $\mathrm{F}$ & 69 & Viuda & No & Líder comunitario & Activo $^{a}$ \\
\hline Sr. $L^{d}$ & M & 71 & Separado & No & Trabajo ocasional & $\begin{array}{l}\text { Lista } \\
\text { espera }^{b}\end{array}$ \\
\hline Sra. AM & $\mathrm{F}$ & 60 & Casada & No & Trabajo ocasional & $\begin{array}{l}\text { Lista } \\
\text { espera }^{b}\end{array}$ \\
\hline Sra. J & $\mathrm{F}$ & 70 & Viuda & No & Recibe pensión & $\begin{array}{l}\text { Clubes de } \\
\text { mayores }^{c}\end{array}$ \\
\hline Sr. JC & M & 64 & Soltero & No & Trabajo ocasional & $\begin{array}{l}\text { Lista } \\
\text { espera }^{\mathrm{b}}\end{array}$ \\
\hline
\end{tabular}

Nota: a Activo en el proyecto: recibe el subsidio mensual de 80 mil pesos colombianos (equivalente a 40 dólares americanos).

${ }^{\mathrm{b}}$ Lista de espera: no recibe el subsidio.

c Participa en la modalidad de clubes y organizaciones de mayores y no recibe el subsidio.

d Víctima de desplazamiento forzado.

\section{Técnicas de producción de datos}

Se llevó a cabo la técnica de entrevista semiestructurada, orientada a identificar el concepto de bienestar y sus posibles determinantes. La pauta de entrevista se construyó en base a temas, basándose en la experiencia de las autoras en el trabajo con población mayor. El análisis de datos se realizó simultáneamente a la producción de estos.
Los temas a tratar en las entrevistas fueron: (a) introducción; (b) percepción general de bienestar y satisfacción con la vida; (c) independencia y autonomía; (d) apoyo social; (e) percepción de salud; (f) espiritualidad y religión; (g) área laboral, ocio y recreación; y $(\mathrm{h})$ ingresos. Las entrevistas se realizaron en un formato flexible, lo que significa que no todos los temas fueron tratados con todos los sujetos (pues para algunas personas no aplicaban) y, a su vez, en algunos casos, la entrevista permitió identificar otros temas relevantes. 


\section{Procedimiento}

Inicialmente se obtuvo el permiso para la realización de las entrevistas por parte de la Secretaría de Integración Social. Luego se inició el reclutamiento de los participantes, quienes fueron contactados vía telefónica y personalmente en algunas Subdirecciones Locales de Bogotá, en donde se le informó a cada uno sobre el objetivo del estudio, el principio de confidencialidad y se obtuvo el consentimiento informado por cada participante. Se estableció una cita con cada uno $\mathrm{y}$ las entrevistas se realizaron en cada Centro Local (instalaciones de la SDIS en cada localidad (comuna) de Bogotá) y algunas en la vivienda de los participantes. La producción de los datos se realizó en el 2009 en Bogotá. Durante el año 2011 se realizó el análisis de los datos usando la metodología de la teoría fundamentada, las entrevistas fueron codificadas siguiendo este enfoque. De estos análisis surgieron 113 categorías iniciales, las cuales fueron agrupadas en ocho categorías principales y 13 categorías secundarias. El proceso de análisis de datos se describe a continuación.

\section{Análisis de los datos}

Como ha sido mencionado anteriormente, el análisis de los datos se llevó a cabo bajo la teoría fundamentada (grounded theory), la cual es una metodología con un enfoque sistemático inductivo y comparativo para el análisis de los datos cualitativos y la construcción de teorías (Charmaz, 2006; Charmaz y Henwood, 2008; Strauss y Corbin, 2002). Este enfoque permite la creación de teoría a partir de los datos, en vez de basarse en constructos, conceptos, categorías o teorías preconcebidas (Charmaz, 2006; Strauss y Corbin, 2002). Strauss y Corbin (2002) afirman que "la teoría fundamentada es una metodología que no solo genera teoría, sino también puede fundamentarla en los datos" (p. 9).

Así, el análisis fue realizado usando el enfoque propuesto por Charmaz (2006). Cada transcripción fue leída y re-leída, y se inició con la codificación línea por línea donde se buscaron acciones, sin salirse del texto ni hacer interpretaciones teóricas. Luego se continuó con la codificación abierta, en donde se identificaron temas comunes, estilos, patrones, consistencias y dife- rencias, las cuales se fueron convirtiendo en categorías iniciales, como lo recomiendan Charmaz (2006). Acá las entrevistas fueron intercambiadas entre las integrantes del equipo investigador para lograr una triangulación entre investigadores.

Se continuó con una codificación axial, donde se sintetizaron y explicaron los datos y luego se buscaron relaciones entre categorías para dar inicio a la creación de subcategorías (Strauss y Corbin, 2002). Para finalizar se realizó la codificación selectiva, en la cual se integraron, sintetizaron y se explicaron los resultados, dando como resultado el modelo de bienestar subjetivo de personas mayores en pobreza específicamente del proyecto. Este modelo fue discutido y construido entre todo el equipo de investigadores. El proceso de análisis se realizó de manera manual, solo el network final se construyó a través de software ATLAS.ti versión 6.

\section{Consideraciones éticas}

Se contó con autorización de la Secretaría Distrital de Integración Social y consentimiento informado verbal por parte de los participantes (registro de audio) a quienes se les informó acerca de la confidencialidad de la información, objetivos, procedimiento, posibles beneficios de la investigación y el derecho a negarse a participar en la misma.

\section{Resultados}

A través del análisis de procesos y de relaciones entre categorías, se pudo identificar que los factores determinates del BS se organizaban en una jerarquía, es decir, que algunas categorías se encontraron con mayor peso que otras. La percepción de control interno es la categoría de mayor jerarquía y es alimentada por seis factores de segundo nivel: (a) la percepción de apoyo social; (b) las actividades productivas; (c) la seguridad económica salud; (d) las fortalezas personales; (e) los factores culturales; y (f) el acceso a información y capacitación. En la figura 1, se presenta el modelo de bienestar con las categorías y sus relaciones.

La percepción de control interno se identificó como una categoría de primer nivel debido a que 
se observó que para todos los participantes era la clave para la percepción de bienestar. Esta categoría se refiere a que las personas mayores buscan tener el control de sus decisiones, acciones y de su ambiente, lo que facilita que logren autonomía. Un ejemplo de esto se ve claramente en lo que afirma la Sra. H:

Es muy importante (el subsidio), porque así no dependo de nadie. A mis 70 años que voy a cumplir, entonces pues me muevo, me muevo sola. Yo no dependo de que mi nuera, de que mi hija haga el almuerzo, porque yo sé que tengo y que puedo hacer.

El bienestar subjetivo de las personas mayores que no contaban con una percepción de control interno y autonomía se veía amenazado. Por ejemplo, una de las personas en situación de dependencia física, manifestaba que su gran deseo era poder realizar actividades por sí sola, sin tener que depender de otros: "Que yo pudiera salir a caminar, como primero que salía por allá sin pedirle favores a nadie" (Sra. MC). La participante $\mathrm{V}$ también asegura: "Lo único que me molesta es que ya no puedo salir sola como antes lo hacía".

Por otro lado, se encontró que percibir apoyo social está relacionado con el BS. El apoyo social era brindado por la familia, amigos y/o el Estado. No se identificó una prioridad especto al tipo de apoyo, el tamaño de la red de apoyo, el tipo de fuente de apoyo ni la frecuencia de contacto. En otras palabras, la percepción de apoyo es lo que se asocia al BS. Por ejemplo, para la Sra. H el apoyo de sus hijos es lo que más valora: “...para mí lo más importante en mi vida son mis hijos", mientras que el Sr. C, resalta la importancia de la relación con sus amigos: "esa costumbre de que uno tiene, voy a encontrarme con fulano de tal aunque sea para hablar y quitarse uno el aburrimiento"; y para la Sra. B, quien nunca tuvo hijos y es de pocos amigos, recibir ayuda del Estado ha generado una percepción de apoyo: "porque para nosotros hemos encontrado como una mamá la señora [funcionaria]... tengo diversión, nos sacan, nos da mucha tristeza cuando ella no puede ir".

A pesar que la percepción de apoyo no se relacionaba con una fuente en particular, se encontró que las distintas fuentes brindan funciones similares, por ejemplo, los amigos y familia brindan compañía, reconocimiento, diversión y apoyo económico. La Sra. H refiere: "Ahí sobrevivo es con lo que se gana mi hija y con lo que me dan a mi acá". En tanto, la Sra. H indica: "yo no soy capaz de vivir sola, yo tengo que vivir con mis hijos".

El apoyo social brinda a su vez reconocimiento de parte de los otros, el cual se logra especialmente a través del apoyo emocional e instrumental, pues permite que los adultos mayores se perciban como personas autónomas y que contribuyan a otros. El Sr. C expresa: "Me gusta servirle a la comunidad, que eso es muy importante y hacerlo de buena voluntad, hacerlo con amor. Uno tiene que estar con las personas, colaborarles lo que más pueda".

La vinculación a actividades que son percibidas como "productivas" también se aprecian como generadoras de bienestar. Las actividades percibidas como productivas hacen referencia a aquellas que generan algún tipo de recompensa (no necesariamente económica), que facilita la experiencia de emociones positivas. Por ejemplo el Sr. $\mathrm{R}$ refiere: "Me siento divinamente, porque por lo menos hay alguien con quien hablar, se divierte uno un poco"; Sr. S también asegura: "tenemos un grupo de tejo ${ }^{2}$... eso me motiva a mí mucho, porque yo dirijo el grupo y me siento feliz porque estoy haciendo una buena labor".

Los resultados mostraron que el estar vinculado a estas actividades alivia a los adultos mayores de las preocupaciones económicas. El Sr. S afirma: "nosotros compartimos nuestras historias, y después nos divertimos mucho y olvidamos toda esa cantidad de cosas que tenemos en la cabeza". A su vez "tener algo que hacer" era característico de las personas que se sentían satisfechas con sus vidas. Por su parte Sra. H reporta:

me levanto, me baño, hago mis ejercicios que le enseñan a uno hacer de rutina, así sea que lo haga uno en un parque, así sea, que los haga uno en la casa, pues me gusta mantener todas las cosas arregladas y después me gusta venirme para acá o salir por ahí a caminar con

\footnotetext{
${ }^{2}$ Deporte tradicional colombiano.
} 
los abuelos o visitar un hijo que tengo en el centro y por eso me siento bien, me siento como en paz con mí misma.

Mientras que no contar con una planificación de actividades genera incertidumbre e insatisfacción, como lo asegura Sr. JC: "cuando usted no hace nada, pasa una semana y no pasa nada... no hay nada por que esperar".

Más aún, se identificó que habían personas que eran líderes comunitarios y que, el pertenecer a esos grupos promueve satisfacción, reconocimiento y brinda oportunidades de capacitación. El participante C dice: "Me siento orgulloso de mí mismo y satisfecho por eso (ser voluntario)", asimismo, Sra. H reporta: "ser dinamizadora es muy importante para mi vida, porque a través de ellos usted aprende muchas cosas, a través de las personas que conozco. Si conozco más personas importante, puedo ayudar más".

Se observaron diferencias entre las personas que estaban vinculadas a alguna actividad placentera y aquellas que no lo estaban. Por ejemplo, el participante que se encontraba en situación de desplazamiento, manifestaba cómo la poca actividad amenazaba con su bienestar, Sr. L: "a mí me está enfermando el no hacer nada ... yo a la edad de 70 años por donde me muevo por donde, enseñado a la finca estar llevando maíz, llevando fríjol, recogiendo café, en una cosa y otra".

El trabajo es una actividad de gran deseo y significado para las personas mayores, especialmente para los hombres y para una de las mujeres solteras. Trabajar les brinda independencia, satisfacción y tranquilidad al poder cumplir con sus obligaciones económicas. Un ejemplo de esto es el caso del Sr. C que argumenta: "está uno ocupando el tiempo en esas cosas iy ganando plata! Claro, entonces eso lo llena a uno de satisfacción y no se siente uno viejo".

Los hombres manifestaban a menudo la necesidad de contar con un ingreso fijo, ya sea producto de subsidio del Estado o de un trabajo estable, para poder cubrir los gastos de su hogar y no sentirse como una carga para sus hijos. El Sr. R indica: "yo recibo dinero, y digo 'bueno qué hubo, que falta esto en la casa, que hay que ir a comprar', pues cuando estoy sin plata eso es una amargura".

Se evidenció que la situación de pobreza de las personas mayores de la presente investigación afecta significativamente el $\mathrm{BS}$, especialmente a aquellos en donde su seguridad habitacional se ve amenazada o perciben incertidumbre hacia la garantía de tenerla, como también a quienes tienen dificultades para acceder a servicios y productos. A pesar de que todos los participantes pertenecen a la categoría "población en situación de pobreza", se encontró que quienes tienen asegurado alguno de los factores descritos anteriormente (i.e., seguridad habitacional, acceso a servicios y productos) indican percibir un mayor BS que las personas que no cuentan con esos recursos.

A pesar que los participantes aseguran que el subsidio es insuficiente, este parece facilitar su independencia y autonomía. Un ejemplo de esto se refleja en los que asegura el Sr. C: "pues porque al menos al momento que está uno sin nada, al menos le llegan uno 80.000 pesitos [40 dólares americanos], bueno, al menos hizo mercado, fue y pagó los servicios, así se quede uno sin un peso".

Así, dentro de la categoría general de "seguridad económica" es posible distinguir dos subcategorías relevantes: seguridad habitacional y seguridad en la alimentación.

Respecto a la seguridad habitacional, esta se refiere a contar con vivienda (propia o de sus hijos) que no genere un costo mensual a fin de disminuir las preocupaciones. Esto se ve reflejado en lo expresado por la Sra. A: "yo vivo más o menos tranquila, no tengo que andar pensando que tengo que pagar el arriendo".

En cambio, se encontró que las personas que no cuentan con una seguridad habitacional, manifiestan una preocupación constante e incertidumbre, lo que afecta su bienestar, por ejemplo, la Sra. MC indica: "yo también creo no me quieren pagar más arriendo, es que ellos también... como allí ellos todavía no tienen su casita arreglada ni nada, ellos están bregando para trabajar ahí en la casa". 
Respecto a la seguridad en la alimentación, se encontró que algunas de las personas no podían acceder a las tres comidas diarias y debían ingerir alimentos no cárnicos, debido a sus bajos ingresos, lo que afecta su bienestar. Los hombres especialmente quisieran poder decidir qué tipo de comida ingerir y no tener que limitarse a lo que les "alcance". Un ejemplo de lo anterior es lo afirmado por el Sr. L:

...tomarse un pocillito de chocolate con un pan, eso es torear completamente el hambre... llegar el almuerzo de pronto un arrocito azulado, que eso no alimenta para nada sin carnita ... estar llevando una situación de estas eso es cosa muy horrible.

Respecto a la categoría salud, las personas mayores parecen interpretarla básicamente como la ausencia de enfermedad discapacitante que afecte su funcionalidad, tanto a nivel básico como instrumental, lo que se relaciona con garantizar autonomía y evitar la dependencia de otros. Esto se ver reflejado en lo que postula la Sra. MC, cuando menciona: "que yo pudiera caminar, que pudiera salir, como salía primero por allá, siempre me pongo triste a veces, hay Dios mío, si yo pudiera salir". La Sra. BI refiere: "estar uno bien, que pueda uno hacer las cosas todo sin ninguna preocupación de que está uno enfermo".

En el análisis de los factores socioculturales relacionados con el BS, se identificó cómo la cultura describe a la población adulta mayor y define los determinantes de su bienestar. El pertenecer a esta cohorte formó creencias específicas de religiosidad, rol de género y actitud hacia la vida y el Estado. Esto moldeó actitudes de gratitud hacia un ser superior, resignación hacia su situación de pobreza y conformismo, lo que permite que, a pesar de su condición, perciban su vida de una manera positiva. Por ejemplo, la Sra. AM, quien no recibe subsidio y es cuidadora de una hija y esposo, dice:

me siento bien pues, porque tengo mi esposo, tengo mi hija enferma, la quiero mucho, la adoro es mi vida y me siento contenta. No pues yo no le pido nada a mi Dios, ya lo que tengo es muy grande.
Se encontró que el tener alguna creencia religiosa, les brinda tranquilidad, compañía y esperanza, como asegura la Sra. J: "la oración es lo que más vale para uno sentirse bien ante Dios; el rezar me da satisfacción".

Por otro lado, se observó una suerte de "conformismo aprendido" frente a las opciones que brinda el Estado, como lo asegura el Sr. C:

pues como se dice, no nos dan más. Y uno no puede estar peleando con la gente porque, pues sabemos que es de nosotros, que debería de ser más, pero a la hora de la verdad hay que tener uno paciencia, ¿por qué puede uno hacer?.

Respecto a los factores personales vinculados al $\mathrm{BS}$, se encontró que algunas personas mayores contaban con características como la autoeficacia, la resiliencia, la tolerancia, la gratitud, una mayor autoestima y algunas características de personalidad (e.g., liderazgo, perseverancia, altruismo y optimismo) que facilitaban la evaluación positiva de sus vidas. Como lo manifiesta el Sr. C:

Pues ahorita mi vejez la he llevado con calma, con buen entusiasmo, con vigor, con alegría. Los años no lo encierran a uno, ni uno tiene que encerrarse en los años. ¡No! al contrario, uno tiene que sacar vigor, sacar espíritu de donde no lo tenga para poder sobrevivir, porque si uno se acompleja, yo he vivido mi vida así.

Por otro lado, respecto a las oportunidades de acceso a la información y capacitación, se encontró que las personas que participan dinámicamente en las actividades del proyecto, que han podido acceder a capacitación y tienen acceso a información, han potenciado sus habilidades y pueden ejercer su autonomía. Un ejemplo de esto es la Sra. H quien indica:

...agradezco mucho a este programa porque por este programa me he capacitado mucho, he aprendido a hablar, a desenvolverme, no me da pena de la gente, no me da pena expresar lo que siento ni lo que soy, gracias a todo lo que nos han enseñado acá. 


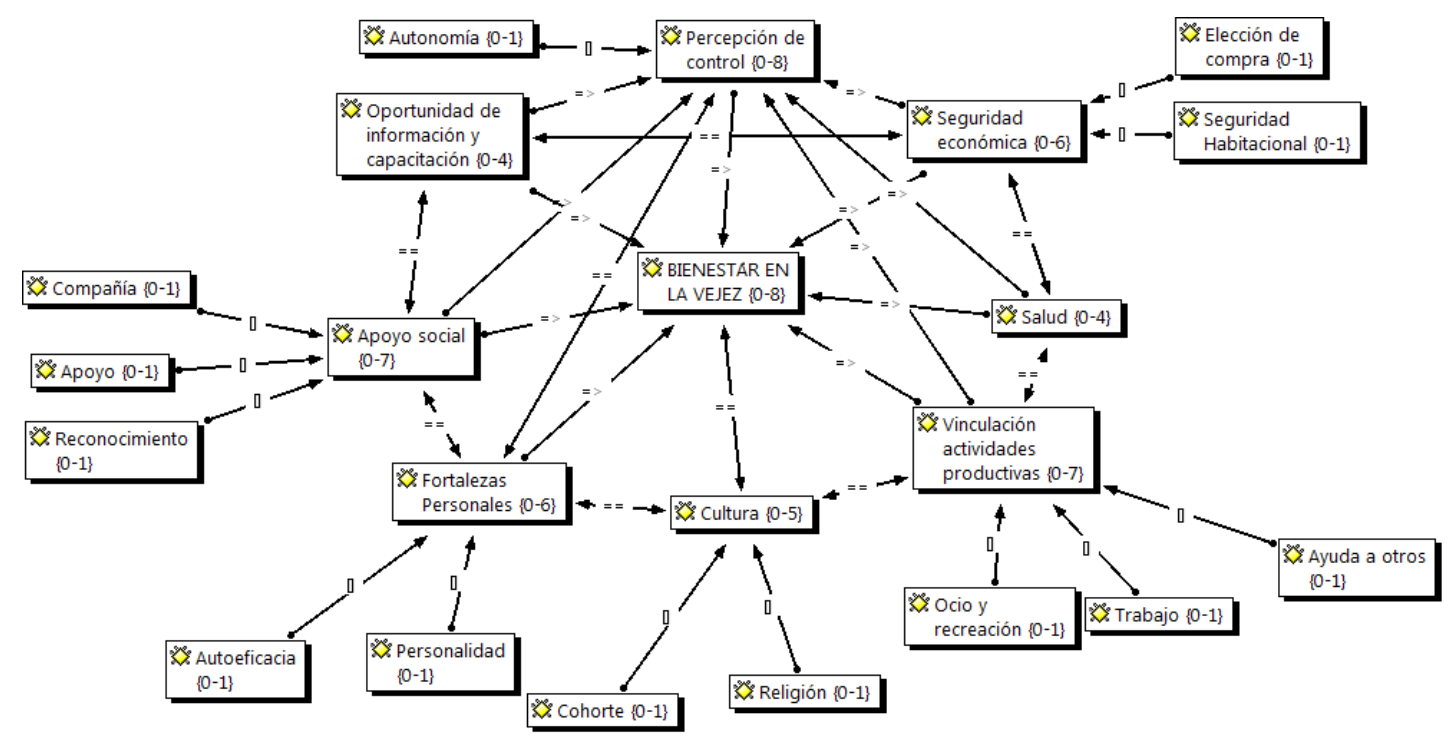

Figura 1. Categorías y relaciones de los determinantes de bienestar subjetivo de las personas mayores en situación de pobreza en Bogotá.

\section{Discusión y conclusiones}

A partir de los resultados se propone un modelo de los determinantes del bienestar subjetivo en la vejez en situación de pobreza, el cual está compuesto por una relación de factores como: la percepción de control interno, el apoyo social, la vinculación a actividades productivas, la seguridad económica, la percepción de la salud, algunos factores culturales, las fortalezas personales y la oportunidad de acceder a información y a instancias de capacitación. El modelo propuesto comparte las categorías de salud y apoyo social percibidas propuesta por Mella et al. (2004), pero propone factores adicionales, conceptualizando y explicitando sus interrelaciones. A su vez, contrario a lo propuesto por Mella et al., en el presente estudio no se encontraron diferencias por sexo.

La presente investigación logró reconocer la importancia de explorar en los factores culturales y generacionales que influyen en los significados que tienen las personas mayores dentro de un contexto social determinado, pues estos influyen directamente en la percepción de bienestar.

Respecto a las dimensiones identificadas, la percepción de control interno, va en la línea de los hallazgos de Bennett, Stenhoff, Pattinson, y Woods (2010), y de Daatland y Hansen (2007), quienes han reportado cómo la percepción de control en la vejez es fundamental para el bienestar y este como promotor de autonomía en la población europea. Así, la percepción de control interno se convierte en un factor valioso durante esta etapa de la vida (Cárdenas y López, 2010; Daatland y Hansen, 2007; Ong y Bergeman, 2004).

Por otro lado, los hallazgos de la presente investigación concuerdan con los realizados por Windle y Woods (2004) quienes han mostrado que los recursos psicológicos son predictores de BS; estos autores encontraron que la relación entre las variables de dificultades físicas y vivienda con el bienestar subjetivo estaba mediada por el control ambiental.

El haber encontrado el apoyo social como factor promotor de bienestar es consistente con lo hallado por otras investigaciones que lo consideran un factor relevante en la vejez (Bennett et al., 2010; Bisconti y Bergerman, 1999; Fernández-Ballesteros, 2009; Ong y Bergeman, 2004), pues ayuda al afrontamiento de situaciones difíciles, a la vinculación a actividades y está relacionado con una mejor calidad de vida (Hildon et al., 2010). 
Los hallazgos respecto a la vinculación en actividades se relacionan con los reportado por Bisconti y Begerman (1999), Fernández-Ballesteros (2009), Glass, Mendes de Leon, Bassuk y Berkman (2006), quienes han mostrado su asociación con tener una mejor salud física y mayores redes sociales, así como su capacidad para mantener y mejorar la capacidad mental y fomentar pensamientos y actitudes positivas.

De manera similar a lo encontrado en este estudio, otras investigaciones (e.g., Bradshaw y Ellison, 2010; Krause, 2003; Krause y Bastida, 2009; Ouwehand et al., 2007) han reportado que las fortalezas personales (e.g., tener un afrontamiento activo), facilitan la percepción de bienestar y que el sentido de religiosidad está asociado a la vejez y facilita el afrontamiento a situaciones adversas.

Tomando en cuenta los antecedentes de investigaciones previas en la temática, consideramos que la presente investigación contribuye a la literatura, entregando una propuesta de un modelo de los determinantes del BS en personas mayores en situación de pobreza pertenecientes a un programa del Estado, constituyendo un primer intento en construir un modelo propio, adaptado y basado a la realidad colombiana.

Pese al aporte que esta investigación ha realizado, es importante notar que sus alcances tienen algunas limitaciones y por tanto, sus resultados deben ser considerados con cautela. Al respecto, es importante notar que la mayoría de los parti- cipantes del presente estudio se encontraba vinculado a un proyecto social de la alcaldía, por lo tanto, los resultados anteriormente expuestos no deben ser generalizados a la población adulta mayor colombiana, que no esté cubierta por este servicio.

Por otro lado, debido a que no fue posible volver a contactar a los participantes para poder profundizar en algunas categorías, en el presente estudio no fue posible lograr una saturación teórica con el fin de lograr generar una teoría sustantiva. Por lo tanto, los resultados y el modelo planteado por este estudio, no deben considerarse como un nuevo modelo teórico probado, sino más bien como una propuesta que debe ser profundizada.

A pesar del alcance limitado del estudio, es importante resaltar que en Colombia no se cuentan con estudios similares y se espera que los hallazgos puedan ser considerados al momento de evaluar y formular políticas públicas.

Proponemos que futuras investigaciones continúen profundizando en el modelo propuesto por esta investigación, por ejemplo, incluyendo a personas mayores que tienen un ingreso fijo, una seguridad habitacional, acceso a los servicios de salud, transporte y alimentación constante, de modo de lograr resultados que permitan una mirada amplia al BS en la población adulta, considerando sus múltiples características de manera integral.

\section{Referencias}

Alcaldía Mayor de Bogotá. (2010). Política pública social para el envejecimiento y la vejez en el Distrito Capital, 2010-225. Bogotá: Autor.

Alonso, L., Ríos, A., Caro de Payares, S., Maldonado, A., Campo, L., Quiñonez, D. y Zapata, Y. (2010). Percepción del envejecimiento y bienestar que tienen los adultos mayores del hogar geriátrico San Camilo de la ciudad de Barranquilla (Colombia). Salud Uninorte, 25(2), 250-259.

Altamar, P. (2010). El concepto y medición de bienes- tar en la evaluación y diseño de programas de transferencias en la vejez. (Tesis doctoral inédita). Facultad Latinoamericana de Ciencias Sociales, México D. F., México.

Benight, C. y Bandura, A. (2004). Social cognitive theory of posttraumatic recovery: The role of perceived self-efficacy. Behavioural research and therapy, 42, 1129-1148. http://dx.doi.org/10.1016/j.brat.2003.08.008

Bennett, K., Stenhoff, A., Pattinson, J. y Woods, F. (2010). "Well if he could see me now": the 
facilitators and barriers to the promotion of instrumental independence following spousal bereavement. Journal of Gerontological Social Work, 53, 215-234.

http://dx.doi.org/10.1080/01634370903562931

Bisconti, T. y Bergeman, C. (1999). Perceived social control as a mediator of the relationships among social support, psychological well-being, and perceived health. The Gerontological Society of America, 39(1), 94-103. http://dx.doi.org/10.1177/0733464805281908

Bradshaw, M. y Ellison, C. G. (2010). Financial hardship and psychological distress: Exploring the buffering effects of religion. Social Science \& Medicine, 71, 196-204.

Cárdenas, A. y López, L. (2010). Analysis matrix of resilience in the face of disability, old age and poverty. International Journal of Disability, Development and Education. 57(2), 175-189.

Charmaz, K. (2006). Grounded theory in the $21^{\text {st }}$ century: Application for social justice studies. En N. Denzin y Y. Lincoln, (Eds.), The SAGE handbook of qualitative research (pp. 359-380). Londres: SAGE.

Charmaz, K. y Henwood, K. (2008). Grounded Theory. En C. Willig y W. Stainton-Rogers, (Eds.), The SAGE handbook of qualitative in psychology (pp. 240-257). Londres: SAGE.

Daatland, S. y Hansen, T. (2007). Wellbeing, control and ageing. En H. Mollenkkopf y A. Walkerm, (Eds.). Quality of life in old age (pp. 33-47). Londres: Springer.

Deci, E. y Ryan, R. (2008). Hedonia, eudaimonia, and well-being: An introduction. Journal of Happiness Studies, 9, 1-11. http://dx.doi.org/10.1007/s10902-006-9018-1

Diener, E., Eunkook, M., Richard, S., Lucas, E. y Smith, H. (1999). Subjective well-being: Three decades of progress. Psychological Bulletin, 125(2), 276-302.

Diener, E., Oishi, S. y Lucas, R. (2003). Personality, culture and subjective well-being: Emotional and cognitive evaluations of life.
Annual Review of Psychology, 54, 403-425.

http://dx.doi.org/10.1146/annurev.psych.5 4.101601.145056

Departamento Administrativo de Bienestar Social. (2006). Manual de procedimientos: proyecto 7217- atención para el bienestar de la persona mayor en pobreza en Bogotá D.C. Bogotá, D.C: Autor.

Fernández-Ballesteros, R. (2009). Psicología de la vejez: una psicogerontologia aplicada. Madrid: Pirámide.

Glass, T., Mendes de Leon, C., Bassuk, S. y Berkman, L. (2006). Social engagement and depressive symptoms in late life: Longitudinal findings. Journal of Aging Health, 18, 604-628. http://dx.doi.org/10.1177/0898264306291017

Hildon, Z., Montgomery, S., Blane, D., Wiggins, R. y Netuveli, G. (2010). Examining resilience of quality of life in the face of health-related and psychosocial adversity at older ages: What is "right" about the way we age? The Gerontologist, 50(1), 36-47. http://dx.doi.org/10.1093/geront/gnp067

Keyes, C., Shmotkin, D. y Ryff, C. (2002). Optimizing well-being: The empirical encounter of two traditions. Journal of Personality and Social Psychology, 82, 1007-1022. http://dx.doi.org/10.1037/00223514.82.6.1007

Krause, N. (2003). Religious meaning and subjective well-being in late life. Journal of Gerontology Series B Social Sciences, 58(3), S160S170. http://dx.doi.org/10.1093/geronb/58.3.S160

Krause, N. y Bastida, E. (2009). Religion, suffering, and health among older Mexican Americans. Journal of Aging Studies, 23(2), 114-123.

http://dx.doi.org/10.1016/j.jaging.2008.11.002

Linting, M., Meulman, J. J., Groenen, P. J. y van der Kooij, A. J. (2007). Stability of nonlinear principal components analysis: An empirical study using the balanced bootstrarp. Psychological Methods, 12(3), 359-379. 
Mella, R., González, L., D'Appolonio, J., Maldonado, I., Fuenzalida A. y Díaz, A. (2004). Factores asociados al bienestar subjetivo en el adulto mayor. Psykhe, 13(1), 79-89.

Merz, E. y Huxhold, O. (2010). Wellbeing depends on social relationship characteristics: Comparing different types and providers of support to older adults. Ageing \& Society, 30, 843-857.

http://dx.doi.org/10.1016/j.jaging.2008.11.002

Nussbaum, M. (2011). Creating capabilities: The buman development approach. Cambridge, MA: Belknap Press of Harvard University Press.

Nussbaum, M. y Sen, A. (1993). The quality of life. Oxford: Oxford Scholarship Online. http://dx.doi.org/10.1093/0198287976.001.0001

Ong, A. y Bergeman, S. (2004). Resilience and adaptation to stress in later life: Empirical perspectives and conceptual implications. Ageing International, 29(3), 219-246.

Ong, A., Bergeman, C. y Boker, S. (2009). Resilience comes with age: Defining feature in later adulthood. Journal of personality, 77(6), 1777-1804.

http://dx.doi.org/10.1111/j.1467-

6494.2009.00600.x

Ouwehand, C., De Ridder, D. y Bensing, J. (2007). A review of successful aging models: Proposing proactive coping as an important additional strategy. Clinical Psychology Review, 27, 873-884.

http://dx.doi.org/10.1016/j.cpr.2006.11.003

Panel on Measuring Subjective Well-Being in a Policy-Relevant Framework, Committee on National Statistics, Division on Behavioral and Social Sciences and Education $y$ National Research Council. (2013). Subjective well-being: Measuring happiness, suffering and other dimensions of experience. Washington, D.C.: The National Academies Press.

Pethtel, O. y Chen, Y. (2010). Cross-Cultural aging in cognitive and affective components of subjective well-being. Psychology and Aging, 25, 725-729. http://dx.doi.org/10.1037/a0018511

Ponce-Zubillaga, M. G. (2013). Pobreza y bienestar: una mirada desde el desarrollo. Cuadernos del CENDES, 30(83), 1-21A.

Profamilia. (2010). Encuesta Nacional de Demografía y Salud. Bogotá: Autor.

Requena, F. (2003). Bienestar subjetivo, redes de apoyo en los jubilados y sistemas de bienestar. Recuperado de

http://www.fes-

web.org/uploads/files/modules/congress/1

0/grupos-trabajo/ponencias/394.pdf

Ryan, R. y Deci, E. (2001). On happines and human potencial: A review of research on hedonic and eudaimonic well-being. Annual Review of Psychology, 52, 141-66.

Ryff, C. y Keyes, C. (1995). The structure of psychological well-being revisited. Journal of Personality and Social Psychology, 69, 719-727.

Secretaría Distrital de Integración Social. (2008). Construcción participativa de política distrital de envejecimiento y vejez. Bogotá, D.C.: Autor.

Secretaría de Integración Social. (2012). Atención integral para personas mayores: disminuyendo la discriminación y la segregación socioeconómica. Bogotá: Alcaldía Mayor de Bogotá. Recuperado de http://old.integracionsocial.gov.co/anexos/docu mentos/2014_proy_boghum/proyecto_742.pdf

Sen, A. (1995). Inequality re-examined. Oxford: Oxford Scholarship Online. http://dx.doi.org/10.1093/0198289286.001.0001

Strauss, A. y Corbin, J. (2002). Bases de la investigación cualitativa: técnicas y procedimientos para desarrollar la teoría fundamentada. Medellín, Colombia: Universidad de Antioquía.

Teo, T. (2009). Philosophical concerns in critical psychology. En D. Fox, I. Prileltensky y S. Austin. S. (Eds.). Critical psychology: An introduction (pp. 36-53). Londres: SAGE.

United Nations Population Fund. (2012). Ageing in the twenty-first century: A celebration 
and a challenge. New York: Autor.

Universidad Nacional de Colombia, Fondo de Población de las Naciones Unidas y Secretaría Distrital de Integración Social. (2009). Linea de base para la formulación de la política de envejecimiento y vejez en Bogotá D.C. Bogotá: Autores.
Vera, J., Sotelo, T. y Domínguez, M. (2005). Bienestar subjetivo, enfrentamiento y redes de apoyo social en adultos mayores. Revista Intercontinental de Psicología y Educación, 7(2), 57-78.

Windle, G. y Woods, R. (2004). Variations in subjective wellbeing: The mediating role of a psychological resource. Ageing and Society, 24, 583-602.

Fecha de recepción: 8 de septiembre de 2014 Fecha de aceptación: 3 de diciembre de 2014 